\title{
Low-energy limit of the three-band model for electrons in a $\mathrm{CuO}_{2}$ plane
}

\author{
V.I.Belinicher and A.L.Chernyshev \\ Institute of Semiconductor Physics, 630090 Novosibirsk, Russia
}

The three-band model with the O-O direct hopping near to unit filling is considered. We present the general procedure of reduction of this model to the low-energy limit. At unit filling the threeband model in the charge-transfer limit is reduced to the Heisenberg model and we calculate the superexchange constant. For the case of the small electron doping the three-band model is reduced to the $t-J$ model and we calculate electron hopping parameters at the nearest and next neighbors. We derive the structure of corrections to the $t-J$ model and calculate their magnitude. The values of the hopping parameters for electron- and hole-doping differ approximately at $40 \%$.

Keywords: $\mathrm{CuO}_{2}$ planes, $t-J$ model, three-band model, hopping parameter. 


\section{Introduction}

Since the discovery of electron-doped superconductors [1] there is growing interest in structure and properties of these systems [2, 3, 国. The electron- and hole-doped high-temperature superconductors (such as $\mathrm{Nd}_{2-x} \mathrm{Ce}_{x} \mathrm{CuO}_{4}$ and $\mathrm{La}_{2-x} \mathrm{Sr}_{x} \mathrm{CuO}_{4}$ ) both have the $\mathrm{CuO}_{2}$ planes with the same structure constant. In spite of the similar structure the experiments show the strong asymmetry in the properties of these compounds. Thus, the critical temperature for $\mathrm{Nd}_{2-x} \mathrm{Ce}_{x} \mathrm{CuO}_{4}$ is only $22 \mathrm{~K}$ that in contrast with $\mathrm{T}_{c}=40 \mathrm{~K}$ for $\mathrm{La}_{2-x} \mathrm{Sr}_{x} \mathrm{CuO}_{4}$ [5]. Also, the doping concentration which destroy the $\mathrm{AF}$ order for electron- and hole-doped systems are $\sim 15 \%$ and $2 \sim 3 \%$ respectively [2, 3]. To study of such asymmetrical phenomena might help to illuminate the nature of superconductivity in these compounds.

Since the structure of the $\mathrm{CuO}_{2}$ plane is the same for these compounds, one can expect that well known three-band Hamiltonian [6] should be suitable for both of them.

In this paper we investigate one electron over unit filling in $\mathrm{CuO}_{2}$ plane. We start from the Emery Hamiltonian [6] with the O-O direct hopping. On the basis of our general approach to the low-energy reduction [7], we obtain the effective single-band Hamiltonian. The equivalence of this single-band Hamiltonian and the $t-J$ model is discussed and the second-order corrections to the $t-t^{\prime}-J$ model are derived. The effective parameters of the $t-t^{\prime}-J$ model for the electron are calculated in the realistic region of Emery model's parameters. Effective hopping is less than in the case of hole-doping. Similar conclusion was obtained in the work by Zhang and Benneman [8], but they used the perturbation theory over the parameters $t_{p d} / U_{d}, t_{p d} /\left(\epsilon_{p}-\epsilon_{d}\right)$ which does not work as it was argued in our previous works [7, 9].

In Sec.II we represent the three-band model in more suitable terms for reduction to the single-band model. We use a three-step procedure. At the first step we introduce the symmetrical and antisymmetrical oxygen operators. At the second step we separate out the onesite Hamiltonian and get its solution. At the third step we represent the primary Hamiltonian in terms of the Hubbard operators which reflect the structure of the solution of the onesite problem. In Sec. III we briefly consider the three-band model at unit filling. Then, we apply the Schrieffer-Wolff transformation [10] for getting the $J$-term of the low-energy Hamiltonian. In Sec. IV we get the effective electron hopping parameters and correction to them. Also, we discuss the accuracy of the $t-t^{\prime}-J$ models for the electron-doped system. Section V presents our conclusions. In Appendix some details of our consideration are given.

\section{Three-band Hamiltonian}

The three-band model with the direct $\mathrm{O}-\mathrm{O}$ hopping is given by the following Hamiltonian [6]

$$
\begin{aligned}
H \quad & =\epsilon_{d} \sum_{l, \alpha} d_{l \alpha}^{+} d_{l \alpha}+\epsilon_{p} \sum_{m, \alpha} p_{m \alpha}^{+} p_{m \alpha} \\
& +U_{d} \sum_{l} d_{l \uparrow}^{+} d_{l \uparrow} d_{l \downarrow}^{+} d_{l \downarrow}+H^{\prime},
\end{aligned}
$$

where $d_{l \alpha}^{+}\left(d_{l \alpha}\right)$ creates (annihilates) the hole in a $\mathrm{Cu} d_{x^{2}-y^{2}}$ state at site $l, p_{m \beta}^{+}\left(p_{m \beta}\right)$ creates (annihilates) the hole in a $\mathrm{O} p_{x(y)}$ state at site $m, \epsilon_{d}$ and $\epsilon_{p}$ are the energies of $\mathrm{Cu}$ and O levels respectively, $U_{d}$ is a intrasite Coulomb repulsion at the copper site.

The hybridization term $H^{\prime}$ is given by

$$
\begin{aligned}
H^{\prime} & =t \sum_{<l m>\alpha}\left(d_{l \alpha}^{+} p_{m \alpha}+\text { H.c. }\right) \\
& -t p \sum_{<m m^{\prime}>\alpha}\left(p_{m \alpha}^{+} p_{m^{\prime} \alpha}+H . c .\right),
\end{aligned}
$$


where $<l m>$ denote the nearest-neighbor $\mathrm{Cu}$ and $\mathrm{O}$ sites, $<m m^{\prime}>$ denote the nearestneighbor $\mathrm{O}$ sites. In Eq. (2) we follow to the sign convention of Ref. 111, 12.

As it was firstly noted by Zhang and Rice [13], it is conveniently to use the orthonormalized oxygen states on the four oxygens around a $\mathrm{Cu}$ site. In our previous work [7] we have represented the Hamiltonian (11), (2) in terms of orthogonal symmetrical and antisymmetrical oxygen cluster states

$$
\begin{aligned}
q_{l} & =\sum_{\mathbf{k}}\left(1+\gamma_{\mathbf{k}}\right)^{-1 / 2}\left(\cos \left(k_{x} / 2\right) p_{\mathbf{k} x}\right. \\
& \left.+\cos \left(k_{y} / 2\right) p_{\mathbf{k} y}\right) \exp \left(-i \mathbf{k r}_{l}\right) \\
\tilde{q}_{l} & =\sum_{\mathbf{k}}\left(1+\gamma_{\mathbf{k}}\right)^{-1 / 2}\left(-\cos \left(k_{y} / 2\right) p_{\mathbf{k} x}\right. \\
& \left.+\cos \left(k_{x} / 2\right) p_{\mathbf{k} y}\right) \exp \left(-i \mathbf{k r}_{l}\right)
\end{aligned}
$$

where summation in Eq. (3) is produced over the Brillouin zone with $\gamma_{\mathbf{k}}=(1 / 2)\left(\cos \left(\mathrm{k}_{x} \mathrm{a}\right)+\right.$ $\left.\cos \left(\mathrm{k}_{y} \mathrm{a}\right)\right)$ and $p_{\mathbf{k} x, y}$ are the Fourier image of $p_{m x, y}$

$$
p_{\mathbf{k} x, y}=\sum_{m \in x, y} p_{m} \exp \left(-i \mathbf{k r}_{m}\right)
$$

The physical reason for introduction of $q_{l}$ and $\tilde{q}_{l}$ states 13 is as follows. The hole at the copper can hope only at the symmetrical combinations of the oxygen states. If we separate out the oxygen states which interact strongly with the copper states, we can solve the problem of determination of the low-energy twohole states or vacuum states at the background of the one-hole states (spins). The states $q_{l}, \tilde{q}_{l}$ (3) are independent at different sites and are ortonormalized. They are very convenient for solving this problem.

The original Hamiltonian (11), (2) in terms of $q_{l}, \tilde{q}_{l}$ takes the form

$$
\begin{aligned}
H_{0} & =\epsilon_{d} \sum_{l, \alpha} d_{l \alpha}^{+} d_{l \alpha}+\epsilon_{p} \sum_{l, \alpha}\left(q_{l \alpha}^{+} q_{l \alpha}+\tilde{q}_{l \alpha}^{+} \tilde{q}_{l \alpha}\right) \\
& +U_{d} \sum_{l} d_{l \uparrow}^{+} d_{l \uparrow} d_{l \downarrow}^{+} d_{l \downarrow}
\end{aligned}
$$

$$
\begin{aligned}
H^{\prime} & =2 t \sum_{<l l^{\prime}>\alpha} \lambda_{l l^{\prime}}\left(d_{l \alpha}^{+} q_{l^{\prime} \alpha}+H . c .\right) \\
& -t_{p} \sum_{<l l^{\prime}>\alpha}\left\{\mu_{l l^{\prime}}\left(q_{l \alpha}^{+} q_{l^{\prime} \alpha}-\tilde{q}_{l \alpha}^{+} \tilde{q}_{l^{\prime} \alpha}\right)\right. \\
& \left.+\nu_{l l^{\prime}}\left(q_{l \alpha}^{+} \tilde{q}_{l^{\prime} \alpha}+H . c\right)\right\}
\end{aligned}
$$

where the explicit form of the coefficients $\lambda_{l l^{\prime}}$, $\mu_{l l^{\prime}}, \nu_{l l^{\prime}}$ can be obtained from Eqs. (11), (2), (3)

$$
\begin{aligned}
& \{\lambda, \mu, \nu\}_{l l^{\prime}} \equiv\{\lambda, \mu, \nu\}\left(\mathbf{l}-\mathbf{l}^{\prime}\right) \\
& =\sum_{\mathbf{k}}\{\lambda, \mu, \nu\}_{\mathbf{k}} \exp \left(-i \mathbf{k}\left(\mathbf{l}-\mathbf{l}^{\prime}\right)\right),
\end{aligned}
$$

with

$$
\begin{aligned}
\lambda_{\mathbf{k}} & =\left(1+\gamma_{\mathbf{k}}\right)^{1 / 2}, \\
\mu_{\mathbf{k}} & =8 \cos ^{2}\left(k_{x} / 2\right) \cos ^{2}\left(k_{y} / 2\right)\left(1+\gamma_{\mathbf{k}}\right)^{-1 / 2}, \\
\nu_{\mathbf{k}} & =4 \cos \left(k_{x} / 2\right) \cos \left(k_{y} / 2\right)\left(\cos ^{2}\left(k_{x} / 2\right)\right. \\
& -\cos ^{2}\left(k_{y} / 2\right)\left(1+\gamma_{\mathbf{k}}\right)^{-1 / 2} .
\end{aligned}
$$

These coefficients decrease rapidly with increasing $\left|\mathbf{l}-\mathbf{l}^{\prime}\right|$. The values of $\lambda, \mu$ and $\nu$ for small $\left|\mathbf{l}-\mathbf{l}^{\prime}\right|$ are given in Table I. One can easily get that the $\nu_{00} \equiv 0$ and, therefore, the mixing of $q$ and $\tilde{q}$ at the same sites is absent. The transformed Hamiltonian (5) is equivalent to the three-band Hamiltonian (1), (2).

We divide the Hamiltonian (5) into local and hopping parts

$$
\begin{aligned}
H_{l o c} & =\epsilon_{d} \sum_{l, \alpha} d_{l \alpha}^{+} d_{l \alpha}+\left(\epsilon_{p}-\mu_{0} t_{p}\right) \sum_{l, \alpha} q_{l \alpha}^{+} q_{l \alpha} \\
& +\left(\epsilon_{p}+\mu_{0} t_{p}\right) \sum_{l, \alpha} \tilde{q}_{l \alpha}^{+} \tilde{q}_{l \alpha} \\
& +U_{d} \sum_{l} d_{l \uparrow}^{+} d_{l \uparrow} d_{l \downarrow}^{+} d_{l \downarrow} \\
& +2 t \lambda_{0} \sum_{l l^{\prime} \alpha}\left(d_{l \alpha}^{+} q_{l \alpha}+H . c .\right) \\
H_{h o p} & =2 t \sum_{l l^{\prime} \alpha} \lambda_{l l^{\prime}}\left(d_{l \alpha}^{+} q_{l^{\prime} \alpha}+H . c .\right) \\
& -t_{p} \sum_{l l^{\prime} \alpha}\left\{\mu_{l l^{\prime}}\left(q_{l \alpha}^{+} q_{l^{\prime} \alpha}-\tilde{q}_{l \alpha}^{+} \tilde{q}_{l^{\prime} \alpha}\right)\right. \\
& \left.+\nu_{l l^{\prime}}\left(q_{l \alpha}^{+} \tilde{q}_{l^{\prime} \alpha}+H . c\right)\right\},
\end{aligned}
$$

hereafter sum over $l, l^{\prime}$ denotes $l \neq l^{\prime}$. One can see that hybridization term in $H_{l o c}(7)$ includes 
only the symmetric oxygen state in agreement with Zhang and Rice 13 . The direct O-O hopping, once taken into account, does not mix local states with the opposite symmetry.

For the case of unit filling there is one hole per unit cell. Therefore, one can introduce the set of one-hole cluster state

$$
\begin{aligned}
& \left|d_{\alpha}>\equiv d_{\alpha}^{+}\right| 0>,\left|q_{\alpha}>\equiv q_{\alpha}^{+}\right| 0> \\
& \left|\tilde{q}_{\alpha}>\equiv \tilde{q}_{\alpha}^{+}\right| 0>
\end{aligned}
$$

and rewrite $H_{l o c}$ in this terms

$$
\begin{aligned}
H_{l o c}^{1} & =\sum_{l, \alpha}\left\{\epsilon_{d} X_{l \alpha}^{d d}+\left(\epsilon_{p}-\mu_{0} t_{p}\right) X_{l \alpha}^{q q}\right. \\
& +\left(\epsilon_{p}+\mu_{0} t_{p}\right) X_{l \alpha}^{\tilde{q} \tilde{q}} \\
& \left.+2 t \lambda_{0}\left(X_{l \alpha}^{d q}+\text { H.c. }\right)\right\}
\end{aligned}
$$

where

$$
X_{l \alpha}^{a b} \equiv\left|a_{l \alpha}><b_{l \alpha}\right|
$$

is the Hubbard operator at the site $l, \alpha=$ $\pm \frac{1}{2}$ is the spin projection. It is convenient to introduce the Hubbard operators because they form the natural basis for description of one-site states. If we introduce also the nondiagonal Hubbard operators we can simply express all operators in our Hamiltonian (7) in their terms. Diagonalization of $H_{l o c}^{1}(9)$ is performed for each site independently. After diagonalization $H_{l o c}^{1}$ is given by

$$
\begin{aligned}
H_{l o c}^{1} & =\sum_{l, \alpha}\left\{\epsilon_{f} X_{l \alpha}^{f f}+\epsilon_{g} X_{l \alpha}^{g g}\right. \\
& \left.+\left(\epsilon_{p}+\mu_{0} t_{p}\right) X_{l \alpha}^{\tilde{q} \tilde{q}}\right\}
\end{aligned}
$$

with

$$
\begin{aligned}
& \left|f_{\alpha}>=U\right| d_{\alpha}>-V \mid q_{\alpha}> \\
& \left|g_{\alpha}>=V\right| d_{\alpha}>+U \mid q_{\alpha}> \\
& U=\left(\left(R_{1}+\tilde{\Delta}\right) / 2 R_{1}\right)^{1 / 2} \\
& V=\left(\left(R_{1}-\tilde{\Delta}\right) / 2 R_{1}\right)^{1 / 2}
\end{aligned}
$$

where $R_{1}=\left(\tilde{\Delta}^{2}+4 t^{2} \lambda_{0}^{2}\right)^{1 / 2}, \tilde{\Delta}=\left(\Delta-\mu_{0} t_{p}\right) / 2$, and $\epsilon_{f, g}=-\Delta_{1} \mp R_{1}, \Delta_{1}=\left(\Delta+\mu_{0} t_{p}\right) / 2$, $\Delta=\epsilon_{p}-\epsilon_{d}$. As discussed in Ref. [7], we assume that at unit filling the groundstate of the $\mathrm{CuO}_{2}$ plane is the low $\mid f \alpha>-$ states on each cluster (the hole on the copper with admixture of symmetrical combination of the hole on the nearest four oxygens) with a virtual transitions at neighbors. Such transitions give the superexchange interaction in the second order of perturbation theory.

The hopping part of the Hamiltonian (7) contains transitions between vacuum, one- and two-hole states at the different sites. For a detailed consideration of this subject see Ref. [7].

\section{Superexchange interaction}

Here we consider only the terms of $H_{h o p}$ that are relevant to intersite interaction

$$
\begin{aligned}
H_{h o p} & =\sum_{l l^{\prime} \alpha \beta, y} F_{l l^{\prime}, \beta}^{y, \alpha}\left\{X_{l}^{y, f \alpha} X_{l^{\prime}}^{0, f \beta}\right. \\
& \left.+X_{l^{\prime}}^{f \beta, 0} X_{l}^{f \alpha, y}\right\},
\end{aligned}
$$

where $y$ is the set of two-hole states, which are five singlets and three triplets (Ref. [7]), $F_{l l^{\prime}, \beta}^{y, \alpha}$ are matrix elements of the Hamiltonian for transition from $\left\{f_{l}, f_{l^{\prime}}\right\}$ to $\left\{y_{l}, 0_{l^{\prime}}\right\}$ states.

By applying the Schrieffer-Wolff transformation to (13) (see Appendix and [7, 10]) one can get

$$
\begin{aligned}
& H_{J}=\sum_{l l^{\prime}}\left(J_{l l^{\prime}} \mathbf{S}_{l} \mathbf{S}_{l^{\prime}}+Y_{l l^{\prime}} \hat{N}_{l} \hat{N}_{l^{\prime}}\right), \\
& \mathbf{S}_{l}=(1 / 2) \sigma_{\alpha \beta} X_{l}^{\alpha \beta}, \quad \hat{N}_{l} \equiv X_{l}^{\uparrow \uparrow}+X_{l}^{\downarrow \downarrow},
\end{aligned}
$$

the expressions for the $J_{l l^{\prime}}$ and $Y_{l l^{\prime}}$ constant are given in Appendix.

Thus, we established that at unit filling the groundstate of the $\mathrm{CuO}_{2}$ plane is the system of local spins which interact antiferromagnetically. Since $J_{l l^{\prime}}$ decreases rapidly with increasing $\left|\mathbf{l}-\mathbf{l}^{\prime}\right|$, we hold only the nearest-neighbor 
term in Eq. (14) and receive the Heisenberg Hamiltonian. One can check that $Y_{\left.<l l^{\prime}\right\rangle} \simeq$ $-\frac{1}{4} J_{<l l^{\prime}>}$ and so

$$
\begin{aligned}
& H_{J}=J \sum_{<l l^{\prime}>}\left(\mathbf{S}_{l} \mathbf{S}_{l^{\prime}}-\frac{1}{4} \hat{N}_{l} \hat{N}_{l^{\prime}}\right) \\
& J \equiv J_{<l l^{\prime}>}
\end{aligned}
$$

The second term in the Hamiltonian (15) can be essential when the system goes away from unit filling.

\section{Electron-doped system}

The doping of the $\mathrm{CuO}_{2}$ plane by the electron is equivalent to removal of a hole from one of the clusters. Hence, in the hole picture the electron is 'zero' or vacuum state. If the hole (spin) is taken away from cluster, the neighboring holes (spins) can hope to the empty site. Thus, the mechanism of the movement of the electron is different from the movement of the added hole. The electron moves as a 'hole-inhole', whereas the hole moves as a local singlet.

\subsection{Hopping Hamiltonian}

From expression for $H_{\text {hop }}$ (7) with definitions (12) and (10) one can easily get the zeroorder electron hopping Hamiltonian

$$
H_{t}=\sum_{l l^{\prime} \alpha} T_{l l^{\prime}} X_{l}^{0, f \alpha} X_{l^{\prime}}^{f \alpha, 0}
$$

with

$$
T_{l l^{\prime}}^{e}=-4 t \lambda_{l l^{\prime}} U V-t_{p} \mu_{l l^{\prime}} V^{2}
$$

The electron hopping Hamiltonian (16) coincides with the hole hopping Hamiltonian derived in works [7, 9]. They correspond to the $t-t^{\prime}-\ldots$ terms of the $t-t^{\prime}-J$ Hamiltonian. But the expression for the hole-singlet hopping is more complicated [7]

$$
\begin{aligned}
T_{l l^{\prime}}^{h} \quad & =2 t \lambda_{l l^{\prime}}\left(\sqrt{2} U_{1} U-W_{1} V\right) \times \\
& \times\left(\sqrt{2} V_{1} V-W_{1} U\right) \\
& -t_{p} \mu_{l l^{\prime}}\left(\sqrt{2} V_{1} V-W_{1} U\right)^{2} / 2
\end{aligned}
$$

where $U$ and $V$ were determined in Eq. (12), $U_{1}, V_{1}, W_{1}$ are the components of eighenfunction of the local singlet.

Thus, we mapped the three-band model for one electron over unit filling to the $t-t^{\prime}-J$ model. The Hamiltonian (15),(16) in terms of the Hubbard operators can be rewritten in a more usual form

$$
\begin{aligned}
H_{t-J} & =t \sum_{<l l^{\prime}>, \alpha} \tilde{c}_{l \alpha}^{+} \tilde{c}_{l^{\prime} \alpha} \\
& +J \sum_{<l l^{\prime}>}\left(\mathbf{S}_{l} \mathbf{S}_{l^{\prime}}-\frac{1}{4} \hat{n}_{l} \hat{n}_{l^{\prime}}\right), \\
\tilde{c}_{l \alpha} & =c_{l \alpha}\left(1-\hat{n}_{l,-\alpha}\right), \quad \tilde{c}_{l \alpha}^{+}=\left(\tilde{c}_{l \alpha}\right)^{+}, \\
\mathbf{S}_{l} & =(1 / 2) c_{l}^{+} \sigma c_{l}, \quad \hat{n}_{l}=\left(c_{l}^{+} \cdot c_{l}\right) .
\end{aligned}
$$

Here $c_{l \alpha}^{+}, c_{l \alpha}$ are the electron creation and annihilation operators.

\subsection{Second-order corrections}

With the help of Scrieffer-Wolff transformation we shall obtain the second-order corrections to the $t-t^{\prime}-J$ Hamiltonian. The corrections to the first hopping parameter will be small and the $t-J$ model may be valid. The correction to the other hopping parameters are not relatively small and, therefore, models with the hopping at the neighbors farther than nearest must be more complicated than the simple $t-t^{\prime}-\ldots$ type.

First of all we shall obtain correction to the energy of the electron. Such correction arises due to the virtual process of hopping of the 
electron at the neighbors with transition in excited state and back. Corresponding Hamiltonian is given in Appendix. The resulting second-order correction is

$$
\delta H_{E}=-\sum_{l l^{\prime}} M_{l l^{\prime}} X_{l}^{00} \hat{N}_{l^{\prime}}
$$

with

$$
\begin{aligned}
M_{l l^{\prime}} & =\frac{\left|T_{l l^{\prime}, f}^{g}\right|^{2}}{\epsilon_{g}-\epsilon_{f}}, \\
T_{l l^{\prime}, f}^{g} & =2 t \lambda_{l l^{\prime}}\left(U^{2}-V^{2}\right) \\
& +t_{p} \mu_{l l^{\prime}} U V .
\end{aligned}
$$

The Hamiltonian $\delta H_{E}(20)$ coincides completely with $\delta H_{E}$ in Ref. [7], but the expression (21) for the $M_{l l^{\prime}}$ is sufficiently more simple than for the hole one.

Corrections to the direct hopping parameters can be obtained by transformation of the Hamiltonian (13). The result is

$$
\begin{gathered}
\delta H_{t}=\sum_{l n l^{\prime} \alpha \beta, y} T_{l n l^{\prime}}^{y}\left\{x_{y} X_{l}^{0, f \alpha} X_{l^{\prime}}^{f \alpha, 0} \hat{N}_{n}\right. \\
\left.+z_{y} X_{l}^{0, f \beta} X_{l^{\prime}}^{f \alpha, 0}\left(\sigma_{\alpha \beta} \mathbf{S}_{n}\right)\right\}
\end{gathered}
$$

where $y$ is the set of two-hole states, $x_{y}=1 / 2$ for singlets and $x_{y}=3 / 4$ for triplets, $z_{y}=1$ for singlets and $z_{y}=-1 / 2$ for triplets. The expressions for $T_{l n l^{\prime}}^{y}$ are given in Appendix. As it was shown, the corrections to the $t-t^{\prime}-$ $J$ Hamiltonian have a complicated nature and depend on the filling ( $\hat{N}$-term) and spin state of the neighbors (S-term). The corrections for the hole single-band model [7] and the electron one are quite different.

\subsection{Quantitative results}

We have argued [7] that our general approach to the low-energy reduction of a threeband model is essentially more correct than the other one, because we construct the perturbation theory over the ratio of the effective hopping parameters between the different local states to the energy gap between them. This ratio for the case of $\mathrm{CuO}_{2}$ plane is of the order of 0.1 . All values of the effective singleband Hamiltonian have been derived for arbitrary parameters of the three-band model, without any assumption about a relationship among $t, \Delta$ and $U_{d}$. In our calculation we take $U_{d}=8.2 \mathrm{eV}, t=1.4 \mathrm{eV}, t_{p}=0.7 \mathrm{eV}$, $U_{p}=V_{p d}=0$ according to the different papers [14, 15, 16]. Also, we have taken experimental value of $J=126 \mathrm{meV}$ [17 and have determined selfconsistently charge-transfer gap to be $\Delta=5.07 \mathrm{eV}$. In Table II we present the following effective parameters of one-band model: the hopping parameters to the first, second and third neighbors; the contribution of the direct O-O hopping; corrections to hopping and energy ; value of the ratio $t_{1} / J$.

We obtain for the electron hopping $\mathrm{t}^{e} \equiv$ $\mathrm{t}_{1} \approx 0.39 \mathrm{eV}$ that in 1.36 times less than the hole hopping [7] $\mathrm{t}^{h} \approx 0.53 \mathrm{eV}$. The role of the direct $\mathrm{O}-\mathrm{O}$ hopping for the electron-doped system is lower than for hole one. Actually, the admixture of the O-state for one hole on a cluster is small, unlike for the hole singlet, where added hole mainly on the oxygen. Thus, the OO hopping contribution to the hopping at the nearest-neighbors for electron $\left(\mathrm{t}_{p 1} / \mathrm{t}_{1}\right)^{e} \approx 20 \%$ and for hole $\left(\mathrm{t}_{p 1} / \mathrm{t}_{1}\right)^{h} \approx 36 \%$.

As for the validity of $t-J, t-t^{\prime}-J$ and other models to the electron-doped system, our conclusions are similar to the case of the holedoped system. Thus, if we hold only the hopping at the nearest-neighbors, corrections to the $t-J$ model on a ferromagnetic background is near to $0.13 \%$ for electrons and $2.5 \%$ for holes, and $t-J$ model is valid. If we are interested in the next-neighbors hopping, it is necessary to include the correction (22) to the effective Hamiltonian. This correction has the complicated structure and does not reduce to the simple direct hopping. 


\section{Conclusion}

We have studied the electron doping of the $\mathrm{CuO}_{2}$ plane in the framework of three-band model. By applying our general procedure of the low-energy reduction to the electron-doped system, we conclude that the $t-J$ model is valid as well as to the hole-doped system. We established some asymmetrical properties. Effective hopping parameter for the electron is in 1.36 times less than the hole one. Corrections to the single-band electron and hole $t-J$ models have the different magnitudes. Such corrections are important for transitions at the next-nearest neighbors.

It is doubtful that the difference in the parameters of one-band model may lead to the drastic difference in behavior of hole-doped and electron-doped systems with doping. The strong asymmetrical dependence of the critical concentration for disappearance of antiferromagnetizm is probably connected with the nature of antiferromagnetic ordering in this systems [18]. In the hole-doped systems antiferromagnetizm has a quasi-two-dimensional character and is associated with $C u$ spins. In the electron-doped systems the contribution of $N d$ or $\operatorname{Pr}$ spins in the antiferromagnetic ordering is essential and three-dimensional effects are more important.

\section{Acknowledgments}

We would like to thank O.P.Sushkov for the helpful discussion. This work was supported by the Program 'Universities of Russia as the centers of fundamental reseaches' and by the Counsel on Superconductivity of Russian Academy of Sciences, Grant No. 90214.

\section{Appendix: The second-order corrections to the hopping process}

For obtaining such corrections we use the Schrieffer-Wolff transformation

$$
\begin{aligned}
H \Rightarrow \tilde{H} & =\exp (-S) H \exp (S), \\
S^{+} & =-S .
\end{aligned}
$$

The first order generator of transformation and the second-order correction are

$$
\left[H_{0}, S\right]=-H^{\prime}, \quad \delta H^{2}=(1 / 2)\left[H^{\prime}, S\right],
$$

for this model $H_{0} \equiv H_{l o c}$ (Eq.(7)). For the derivation of $J$-term $H^{\prime} \equiv H_{h o p}$ (Eq.(13)). Expressions for the $J_{l l^{\prime}}$ and $Y_{l l^{\prime}}$ integrals are given by

$$
\begin{aligned}
J_{l l^{\prime}} & =\sum_{y} x_{y} \frac{\left|F_{l l^{\prime}}^{y}\right|^{2}}{\epsilon_{y}+\epsilon_{0}-2 \epsilon_{f}}, \\
Y_{l l^{\prime}} & =-\sum_{y} z_{y} \frac{\left|F_{l l^{\prime}}^{y}\right|^{2}}{\epsilon_{y}+\epsilon_{0}-2 \epsilon_{f}},
\end{aligned}
$$

where $y$ is the set of two-hole states at a cluster, $\epsilon_{y}$ are there eighenenergies, $\epsilon_{0} \equiv 0$ is the energy of vacuum state. Values $x_{y}=2, z_{y}=1 / 2$ for singlets and $x_{y}=-1, z_{y}=3 / 4$ for triplets. Matrix elements $F_{l l^{\prime}}^{y}$ are obtained in Ref. [7]. One can check that the major contribution to $J$ and $Y$ is originated from the matrix element of the transition to the lowest singlet and so $Y_{<l l^{\prime}>} \simeq \frac{1}{4} J_{<l l^{\prime}>}$.

The terms of $H_{\text {hop }}$ (17), which lead to the energy correction, are given by

$$
\begin{aligned}
H_{E}^{\prime} & =\sum_{l l^{\prime} \alpha} F_{l l^{\prime}, f}^{g}\left\{X_{l}^{0, f \alpha} X_{l^{\prime}}^{g \alpha, 0}\right. \\
& \left.+X_{l^{\prime}}^{0, g \alpha} X_{l}^{f \alpha, 0}\right\} .
\end{aligned}
$$

Corresponding generator of the transformation is

$$
\begin{aligned}
S_{E} & =-\sum_{l l^{\prime} \alpha} \frac{F_{l l^{\prime}, f}^{g}}{\epsilon_{g}-\epsilon_{f}}\left\{X_{l}^{0, f \alpha} X_{l^{\prime}}^{g \alpha, 0}\right. \\
& \left.-X_{l^{\prime}}^{0, g \alpha} X_{l}^{f \alpha, 0}\right\}
\end{aligned}
$$


where $\mid f_{\alpha}>$ and $\mid g_{\alpha}>$ are the one-hole states (12) with their energies $\epsilon_{f}$ and $\epsilon_{g}$. Thus, the second-order correction to the energy has the form (20).

Corrections to the direct hopping (22) can be obtained from the transformation of the Hamiltonian (13). Magnitudes $T_{l n l^{\prime}}^{y}$ are given by

$$
T_{l n l^{\prime}}^{y}=\frac{F_{l n}^{y} F_{n l^{\prime}}^{y}}{\epsilon_{y}+\epsilon_{0}-2 \epsilon_{f}} .
$$

The expressions of $F_{l n}^{y}$ were obtained analytically and computed for definite values of parameters of the three-band model in Ref. [7].

\section{References}

[1] H.Takagi, S.Uchida and Y.Tokura, Phys. Rev. Lett. 62 (1989) 1197.

[2] T.R.Thurston et al., Phys. Rev. Lett. 65 (1990) 263.

[3] G.M.Luke et al., Phys. Rev. B 42 (1990) 7981.

[4] Jian Ping Lu and Qimiao Si, Phys. Rev. B 42 (1990) 950.

[5] J.G.Bendors and K.A.Müller, Z. Phys. B 64 (1986) 189.

[6] V.J.Emery, Phys. Rev. Lett. 58 (1987) 2794; C.M.Varma, S.Schmitt-Rink and E.Abrahams, Solid State Commun. 62 (1987) 681.

[7] V.I.Belinicher and A.L.Chernyshev, (to be published).

[8] Weiyi Zhang and K.Bennemann, Phys. Rev. B 45 (1992) 12487.

[9] V.I.Belinicher and A.L.Chernyshev, Phys. Rev. B 46 (1992) ....

[10] G.L.Bir and G.E.Pikus, Symmetry and deformational effects in Semiconductors (Wiley, New York, 1974).

[11] V.J.Emery and G.Reiter, Phys.Rev. B 38 (1988) 4547.

[12] P.M.Frenkel and R.J.Gooding, B.I.Shraiman, and E.D. Siggia, Phys.Rev. B 41 (1990) 350.

[13] F.C.Zhang and T.M.Rice, Phys. Rev. B 37 (1988) 3759.

[14] W.E.Pickett, Rev.Mod.Phys. 61 (1989) 433.

[15] V.V.Flambaum and O.P.Sushkov, Physica C 175 (1991) 347. 
[16] J.Fink at al., Physica C 185-189 (1991) 45.

[17] S.Maekawa, Y.Ohita, and T.Tohyama, Physica C 185-189 (1991) 168.

[18] M.Matsuda, Y.Endoh, and K.Yamada, H.Kojima and I.Tanaka, R.J.Birgeneau and M.A.Kastner, G.Shirane, Phys. Rev. B 45 (1992) 12548. 
Table I.

The values of the coefficients $\lambda\left(\mathbf{l}-\mathbf{l}^{\prime}\right), \mu\left(\mathbf{l}-\mathbf{l}^{\prime}\right), \nu\left(\mathbf{l}-\mathbf{l}^{\prime}\right)$ as functions of $\left(\mathbf{l}-\mathbf{l}^{\prime}\right)=n \mathbf{x}+m \mathbf{y}$.

\begin{tabular}{cccc}
\hline $\mathrm{n}, \mathrm{m}$ & $\lambda_{n, m}=\lambda_{m, n}$ & $\mu_{n, m}=\mu_{m, n}$ & $\nu_{n, m}=-\nu_{m, n}$ \\
& & & \\
\hline & & & \\
0,0 & 0.9581 & 1.4567 & 0.0 \\
1,0 & 0.1401 & 0.5497 & 0.2678 \\
1,1 & -0.0235 & 0.2483 & 0.0 \\
2,0 & -0.0137 & -0.1245 & 0.0812 \\
2,1 & 0.0069 & -0.0322 & 0.0609 \\
2,2 & 0.0035 & 0.0231 & 0.0 \\
& & & \\
\hline
\end{tabular}

Table II.

The first three hopping parameters, the contributions of the direct $\mathrm{O}-\mathrm{O}$ hopping to them, second-order corrections to them on a ferromagnetic background, second-order corrections to the energy, and the ratio $t_{1} / J$.

\begin{tabular}{cccc}
\hline $\begin{array}{c}\text { neighbor } \\
\text { number, } \mathrm{n}\end{array}$ & $\begin{array}{c}\text { direct hopping } \\
t_{n}^{\text {eff }},(\mathrm{eV})\end{array}$ & $\begin{array}{c}\text { direct O-O hopping } \\
t_{p n}^{e f f},(\mathrm{eV})\end{array}$ & $\begin{array}{c}\text { Corrections } \\
\delta t_{n}^{e f f},(\%)\end{array}$ \\
\hline & & & \\
1 & -0.3896 & -0.0765 & 0.125 \\
2 & 0.0180 & -0.0345 & 122.5 \\
3 & 0.0480 & 0.0173 & 24.2 \\
\hline & & & 3.081 \\
Correction to & & Ratio & \\
the energy (eV) & -0.0931 & $\left|t_{1} / J\right|$ & \\
\hline
\end{tabular}

